\title{
La provisión de servicios de salud en Chile: aspectos históricos, dilemas y perspectivas
}

The provision of health services in Chile: historical aspects, dilemmas and prospects

\section{Antonio Carlos Azevedo}




\title{
Atualização
}

Current Comments

\section{La provisión de servicios de salud en Chile: aspectos históricos, dilemas y perspectivas}

\section{The provision of health services in Chile: historical aspects, dilemmas and prospects}

\author{
Antonio Carlos Azevedo \\ Organización Panamericana de la Salud en Chile. Santiago, Chile
}

\begin{abstract}
Resumen
Se hace un breve análisis histórico del Sistema de Salud en Chile, desde el Sistema Nacional de Salud, con énfasis a las reformas ocurridas a partir de 1980, durante el régimen militar y los cambios introducidos desde 1990 por los gobiernos democráticos. Se compara la oferta actual de servicos por el subsistema público com aquella del privado utilizando como indicadores por mil beneficiarios: la tasa de consultas, de exámenes de laboratorio, de hospitalizaciones, de altas y de cirugías y la satisfación usuaria. Se analizan los principales dilemas del sistema de salud y las perspectivas de solución a la luz de las principales medidas tomadas en el presente. Hay buenas perpectivas de que el subsistema público mantenga su liderazgo histórico en la conducción y oferta de servicios en Chile debido a la rigurosa lógica geográfica y demográfica en la oferta de servicios, la disponibilidad estable de recursos financieros, una red de servicios suficiente y además, la considerable motivación de sus recursos humanos. Son mencionados aspectos innovadores que pueden ser útiles a otros sistemas, especialmente en América Latina, tales como: organización y gestión de servicios regionales de salud; metodologías innovadoras de pago de servicios; sistema de vigilancia sanitaria y la regulamentación estatal del financiamento privado en salud.
\end{abstract}

Sistema de salud, organización e administración. Investigación sobre servicios de salud. Extensión de cobertura.

\begin{abstract}
The evolution of the health system in Chile, since its beginning and during the National Health Service period is discribed. The transfornations that this system underwent from 1980 on, during the military regime, and the changes introduced by the democratic governments from 1990 on, with the purpose of
\end{abstract}

Correspondencia para / Correspondence to: Antonio Carlos de Azevedo - Monjitas 689/51 Santiago, Chile.

E-mail: azevedo@paho.org

Recibido en 4.9.1996. Reapresentado en 23.9.1997. Aprobado en 21.10.1997. 
correcting distortions introduced in the previuos period, are commented on. The production of the health services is compared as between the public and private sub-sectors. The indicators used are outpatient attendance per thousand insured inhabitants, use of, lab texts on the same populational basis, hospitalization rates, discharges per thousand insured and surgery rates per thousand. Differences between the two sub-systems are commented on as also are those related to user satisfaction in the two sub-systems. The chief challenges to the public system are considered as well as their possible responses to them on the basis of current policies and the solutions implemented. The present situation in the Public/Private mix in health care in Chile gives rise to the positive expectation that the public subsystem may succed in maintaining its historical leadership in the orientation and health services offered in this country. This positive view is supported by the rigorous geographic and demographic logic of the public subsystem, the stable availability of financial resources (result of the 7\% contribution for health applied on all salaries, complemented by tax funds), a not completely modern but reliable network of public health facilities and the considerable motivation shown by its statt. Some features of the Chilean health system's development are mentioned as potentially useful to the development of health systems in other countries, especially in Latin America, such as organization and management of regional health services, innovative methodologies in health service payment (diagnosed related payment, management agreements and per capita financing for primary care); the health surveilance system (especially for food and drugs) and the state regulation of private health care financers.

Health system, organization and administration. Health services research. Extension of health services coverage.

\section{Resumo}

Realiza-se breve análise histórica do sistema de saúde no Chile, a partir do Serviço Nacional de Saúde, com ênfase nas reformas ocorridas desde 1980,durante o regime militar, e as mudanças introduzidas a partir de 1990 pelos governos democráticos. Compara a oferta atual de serviços pelo sistema público com a do setor privado utilizando como indicadores por mil beneficiários: a taxa de consultas, de exames de laboratório, de hospitalização, de altas e de cirurgias e a satisfação dos usuários. Analisa os principais dilemas do sistema de saúde e suas perspectivas à luz das medidas presentemente tomadas. Há boas perspectivas de que o subsistema público mantenha sua liderança histórica na condução e oferta de serviços no Chile devido à rigorosa lógica geográfica e demográfica na prestação de serviços, a disponibilidade estável de recursos financeiros, uma rede de serviços suficiente e ainda a considerável motivação de seus recursos humanos. São mencionados aspectos inovadores que podem ser aproveitados por outros sistemas, especialmente na América Latina, como: organização e gestão de serviços regionais de saúde, metodologias inovadoras de remuneração de serviços, sistema de vigilância sanitária e a regulamentação estatal do financiamento privado em saúde.

Sistema de saúde, organização e administração. Pesquisa sobre serviços de saúde. Extensão de cobertura.

\section{INTRODUCCIÓN}

Los sistemas de salud, practicamente en todos los países del mundo, pasan por profundas transformaciones. A ese conjunto muy desuniforme de procesos se ha convencionado llamar de "Reforma de los Sistemas de Salud ${ }^{11}$ ". De hecho, se trata de un proceso de adaptación a transformaciones que ocurren en el perfil de las poblaciones y en la realidad nosológica, política y social de los distintos países. Del punto de vista de las poblaciones, el principal fenómeno observado es el acelerado proceso de envejecimiento. En relación a la nosología se observa una transición epidemiológica en distinta intensidad 
pero que afecta a practicamente todos los países desarrollados o no. Afuera el crecimiento en importancia de las enfermedades crónicodegenerativas, los cánceres y los accidentes y violéncias surge una série de patologías de perfil muy complejo, cuyo diagnóstico y planteamiento escapan en general a la capacidad de los sistemas de salud. En este último grupo se encuentran la drogadicción, los problemas de la adolescencia, la violéncia urbana, y bajo muchos aspectos, el SIDA. La Reforma de los Sistemas de Salud se hace necesaria principalmente para dar cuenta de ese nuevo perfil de salud que en algunos países como Chile ocurre practicamente en toda la extensión del País, en otros, como Brasil, es más acentuado en los grandes centros urbanos y en los Estados más desarrollados.

Los presentes comentarios intentan componer una percepción de la evolución reciente del Sistema de Salud de Chile, asimismo de las perspectivas de su evolución en los próximos años y de como esa percepción puede ser útil a otros países de America Latina.

\section{ASPECTOS HISTÓ RICO S DE LA ATENCIÓN DE LA SALUD EN CHILE}

\subsection{El Servicio Nacional de Salud (SNS)}

La mayoría de los autores que analizan los importantes logros de la salud pública chilena, coinciden en relacionarlos no solamente a las bondades aisladas de su sistema de salud pero, además, a una série de políticas públicas que se implementaron en el país a partir de los años 20. En ese período se observa la articulación de una série de políticas sociales, interpretadas como la reacción del capitalismo emergente a los riesgos representados por los movimientos sociales observados en Europa y que han acompañado y seguido a la Primera Guerra Mundial. Ese momento marca la constitución de lo que muchos autores chilenos denominan el Estado Asistencial o Estado Benefactor. De este período es la Ley de Seguros de Enfermedad, Invalidez y Viejez ${ }^{2}$. A partir de 1901 funciona en Chile una red de establecimientos beneficentes denominados "Gotas de Leche" que se preocupaba con la suplementación alimentária a los niños de familias de bajo ingreso ${ }^{4}$.

Esa iniciativa se consolidó más tarde en la denominada Ley de Madre y Niño, de 1939. Esta representó una política de suplementación alimentária muy efectiva y continuada que logro así como otros avances sociales del País atravesar régimes tán disparejos como el Gobierno de la Unidad Popular cuando tuvo gran auge, y el Gobierno Militar. Un otro fenómeno que no todos los autores mencionan como condicionante de la excelente situación de salud que el País paso a exhibir fue el esfuerzo educativo, acelerado después de la Reforma Educacional de 1928, acto que consolidaba una preocupación con la enseñanza que el País mostró desde la época de la independencia (El Instituto Nacional y después U. de Chile, son de 1813). Esa sólida y sostenida iniciativa de educación básica, garantiza no solo la erradicación en términos prácticos del analfabetismo, como principalmente una cobertura educacional muy pareja en relación a los distintos extractos de renta. La Encuesta Casen (muestra domiciliar) mostraba para 1990 que la cobertura de educación básica para el decil inferior de ingreso era de $94,8 \%$ en cuanto para el decil superior era de 99,4\% (promedio total: $96,7 \%$ ). Diferencias reales con relación al ingreso se observan apenas a nivel de la educación media (cobertura para el decil superior del $96,0 \%$ y para el inferior del $64,6 \%)^{10}$.

Otro factor relevante para el éxito del futuro del Servicio Nacional de Salud fue la inexistencia de la duplicidad Seguro Social/Ministerio de Salud, casi que una constante en otros países Latinoamericanos. Cuando se promulgó la mencionada Ley de Seguro Obrero que instituye el seguro social en Chile, la nueva institución encontró las instituciones de beneficencias congregadas (desde 1890, Horwitz ${ }^{4}$, 1995) bajo una Junta Central de Beneficencia lo que permitió que esta institución asumiera en gran parte, la ejecución de los encargos de salud del seguro social (Horwitz², na linha 1995).

A partir de 1952, Chile desarrolló un Sistema de Salud estatal, centralizado, de alta cobertura y al cual muchos historiadores y analistas de salud atribuyen un gran número de logros entre los cuales un descenso acentuado de la mortalidad infantil (del 136,2 por mil en 1950 al 31,8 por mil en 1980), el incremento de la esperanza de vida (53/56,8 años en 1950 al $67,6 / 74,6$ en 1980) ${ }^{2}$, el control de la tuberculosis y la erradicación de la poliomielitis.

Quizás lo más importante es que fue un sistema de cobertura universal, equitativo y de calidad.

\subsection{La Reforma de 1980}

A partir de 1973, Chile se vió bajo un régimen militar que orientó al País hacia un sistema económico y social de naturaleza privatizante y 
monetarista que resultó en cambios significativos en lo relativo a la salud. Estos cambios involucraron:

- La creación de 27 Servicios Regionales de Salud, con considerable grado de autonomía financiera y operacional. Al Ministerio de Salud queda reservado un rol esencialmente normativo.

- Se crea el Fondo Nacional de Salud (FONASA) que administra recursos anteriormente operados por el SNS y además se instituye el sistema de libre elección, con recursos del descuento de $7 \%$ de los sueldos de los trabajadores.

- Municipalización de la Atención Primaria (traspaso de la mayoría de los consultorios de atención primaria a la administración de los municipios).

- Institución (1981) del sistema de salud previsional (ISAPRES) encargado de administrar, por opción voluntaria de los interesados, el $7 \%$ descontado de los salarios.

Un hecho tardío de la Reforma de 1980, pero que la complementa, es la creación en 1991 de la Superintendencia de ISAPRES entidad gubernamental encargada de fiscalizar y orientar las acciones de las ISAPRES en relación a sus clientes.

Ese conjunto de reformas se hizo acompañar por una drástica reducción de la inversión en salud, lo que implicó grave deterioro principalmente de los hospitales, debido a la mayor demanda de este tipo de establecimientos por acciones de mantenimiento. Ese deterioro es mucho más marcado a nivel del área metropolitana que del interior del País.

Otra consecuencia importante de la creación de un sistema de financiamiento privado de las actividades de salud fue el incremento de la red privada de clínicas y una serie de subsidios cruzados, representados por la atención de los hospitales públicos a pacientes del sistema ISAPRES, e incentivos fiscales a eventuales contribuciones de empresas para las cotizaciones de sus empleados, entre otros.

\subsection{Los $\mathrm{G}$ obiemos $\mathrm{D}$ emocráticos - la Situación Actual}

A partir de 1990, los gobiernos democráticos, encontraron el Sistema Nacional de Servicios de Salud consolidado en lo relativo al nuevo rol del sector privado, la municipalización semiimplementada y los servicios públicos deteriorados.

Los esfuerzos que se observan relativos a la recuperación y normalización de las actividades del Sector pueden así ser resumidos:

- Inversión en la recuperación física del sistema (un total de cerca de US\$ 450 millones en 5 años).
Esos recursos se han destinado a la construcción de 3 hospitales nuevos, pero principalmente a la operacionalización de Centros de Apoyo Diagnóstico en algunos sistemas regionales y Centros Diagnóstico y Terapéuticos - CDTs - unidades de atención secundaria resolutiva.

- Apoyo al desarrollo organizacional de los servicios municipalizados de salud. Esta acción se concretó por distintos medios, como el proyecto de Salud Rural, destinado a la mejora de los servicios en comunas más dispersas y el apoyo de las Divisiones de Atención Primaria y Divisiones de Programación al desarrollo de tales servicios.

- Aprobación de la Ley de ISAPRES mejorando el control gubernamental sobre este tipo de institución y controlando los subsidios cruzados. Medidas administrativas, como a disposición de los hospitales públicos del listado de asegurados proporcionada por la Superintendencia de ISAPRES, han contribuido para un mejor control de los subsidios cruzados.

- Acciones de disminución de la Inequidad, no solamente estudios para caracterizar el fenómeno como inversiones específicas de salud en las comunas mas carentes ( 70 de las 330 existentes). Ese esfuerzo se articula con acciones más amplias del gobierno en el combate a la pobreza. Durante el gobierno democrático fue posible reducir la población en estado de extrema pobreza en 995 mil habitantes (cerca del 7,1\% de ese extracto) y la población pobre en 2,14 millones de habitantes $(15,3 \%$ de ese extracto $)^{8}$.

- Estímulo a la participación social. Estas medidas a nivel del Sector Salud, involucran la creación de Comités de Participación a nivel de los Servicios Regionales y de los establecimientos de salud (meta: 70 de éstos hasta el final de 1995).

- Diversificación y racionalización de los mecanismos de remuneración de los servicios: pago per capita, pago asociado a diagnóstico y compromisos de gestión.

- Modernización Administrativa de los Servicios Regionales de Salud.

\section{O FERTA ACTUAL DE SERVICIOSY CALID AD PERCIBIDA EN LOSDOS SU BSISTEMAS}

La participación público-privada actual en Chile, resultado de los procesos descritos arriba se puede caracterizar como correspondiendo a una proporción poblacional de $69,25 \%$ público para $25,8 \%$ privado ISAPREs y cerca de $5 \%$ privado propiamente tal. 
En relación a la disponibilidad de servicios, de las 42.684 camas existentes en el País, 81,4\% pertenecían al sub-sector público (74\% en el Sistema Nacional de Servicios de Salud) ${ }^{3}$. En relación a los gastos per cápita, los del sector privado son 502 más elevados de que los del sector público (alrededor de US\$ 235 contra los US\$ 156 del sector público). Cuando se analiza, entretanto, la pauta de oferta de servicios, las ISAPRES abiertas presentan una tasa de egresos por mil beneficiarios, y de exámenes de laboratorio abajo de las del sector público. Las ISAPRES cerradas a su vez ofrecen una densidad de servicios más elevada que las abiertas pero todavía así, apenas poco más elevada que el servicio público en lo relacionado a exámenes de diagnóstico por imagen (Tabla). La proporción de consultas por beneficiário es bastante semejante entre el SNSS y las ISAPRES abiertas si se acrecentan a las consultas del primero, las realizadas por personal no-médico. Igual ocurre con la tasa de hospitalización por cien consultas, bastante semejante entre los tres sistemas si considero en su conjunto las consultas profesionales y no profesionales en el sub-sector público. Una situación muy semejante, o sea una oferta pareja de servicios entre los dos subsistemas se observa con relación a intervenciones quirúrgicas por mil beneficiarios mostrándose parejas la oferta entre el servicio público y las ISAPRES abiertas existiendo una oferta mas que el doble en las ISAPRES cerradas. Algunas de éstas, como las vinculadas al sistema del cobre, por ejemplo, presentan estándares de gasto per cápita semejantes a países desarrollados (aprox. US\$ 1,000 por beneficiario al año).

En relación a la calidad percibida, se observa un nivel muy elevado de insatisfacción que llega al 55\% para la clentela pública (Sist. FONASA), pero que no se encuentra en niveles muy inferiores para la clientela del subsistema ISAPRES ( $45 \%$ en la misma encuesta). Las personas que efectivamente habían utilizado el sistema o que habían acompañado algún familiar, al ser preguntadas sobre el punto del sistema que les pareció más deficiente, han manifiesto como peor punto el conseguir consulta y la sala de espera (30\% de evaluaciones malo y muy malo para el subsistema público y $8 \%$ para el privado), seguido de la atención por personal administrativo (19\% de malo y muy malo para el subsistema público y $9 \%$ para el privado) del trato de enfermeras y auxiliares (15\% de malo y muy malo para el subsist. público y $5 \%$ para el privado) siendo preservada la atención médica (considerada mala y muy mala por $10 \%$ de los clientes del subsist. público y $3 \%$ del privado) $)^{1,11}$.

En conclusión, el análisis de oferta de servicios por los distintos componentes de los subsistemas de salud conduce a la percepción de un cierto equilíbrio, con diferencias relativamente discretas entre ellos. Llama la tención delante de ese análisis objetivo, el porqué de la existencia, por ejemplo, de listas de espera para cirugía eletiva en el subsistema público, en cuanto en el privado el fenómeno o no existe o no asume importáncia. Una de las explicaciones sería

Tabla - Algunos indicadores de utilización de servicios de salud en Chile* año 1994.

Table - Some indicators of the use of health services in Chile.

\begin{tabular}{|c|c|c|c|}
\hline \multirow[t]{2}{*}{ Indicadores } & \multirow{2}{*}{$\begin{array}{c}\text { Sistema nacional de servicios } \\
\text { de salud (1) }\end{array}$} & \multicolumn{2}{|c|}{ ISAPRES* (2) } \\
\hline & & Abiertas & Cerradas \\
\hline Beneficiarios (en miles) & $8,874(3)$ & $3.479,4$ & 190,5 \\
\hline Consultas médicas (por beneficiario) & $1,71(3,4)(4)$ & 2,99 & 5,04 \\
\hline Electivas $(\%)$ & 65,8 & - & - \\
\hline U rgencia (\%) & 34,2 & - & - \\
\hline Consulta (p/personal p/médico) & 1,69 & - & - \\
\hline \multicolumn{4}{|l|}{$\begin{array}{l}\text { Apoyo diagnóstico } \\
\text { (por consulta): }\end{array}$} \\
\hline - Imagen & $0,173(5)$ & 0,185 & 0,197 \\
\hline - A. Patológica & 0,054 & 0,0345 & 0,0404 \\
\hline - Laboratorio & 1,830 & 0,817 & 0,994 \\
\hline Tasa hospitalización ( $\mathrm{p} / 100$ consultas) & $7,23(2,88)(4)$ & 3,19 & 8,34 \\
\hline Promedio de estadía (en días) & $7,1(6)$ & 3,18 & 4,55 \\
\hline Egresos ( $p / m i l$ beneficiados) & 123,40 & 97,9 & 408,9 \\
\hline Intervenciones quirúrgicas ( $\mathrm{p} / \mathrm{mil}$ beneficiados) & 82,0 & 79,8 & 186,7 \\
\hline Gastos per cápita US\$ & 155,7 & 235,3 & 301,7 \\
\hline $\begin{array}{l}\text { Fuentes: } \\
\text { 1) Ministerio de Salud. Tarjeta de Presentación 1995, Santiago, } 199 \\
\text { 2) Superintendencia de Instituciones de Salud Previsional (ISAPRES) } \\
\text { (3) Corresponde al } 63,3 \% \text { de la población del país. } \\
\text { (4) Calculado en función del total de atenciones (Médicas y Paramé } \\
\text { (5) Datos del Depto. de Coordinación e Informática, Ministerio de S } \\
\text { (6) Datos del D epto. de Coordinación e Informática, Ministerio de S }\end{array}$ & $\begin{array}{l}\text { 5. } \\
\text { ). Boletín Estadístico del Sistema ISAPRE, } \\
\text { Sédicas). } \\
\text { Salud, } 1994 . \\
\text { Salud, } 1993 .\end{array}$ & & \\
\hline
\end{tabular}


la existencia de pacientes del subsistema privado (principalmente ISAPRES abiertas) que se hacen atender por el sistema público. Ese tipo de fenómeno, como ya se ha mencionado, ha disminuido a partir de mediados de 1994 cuando los servicios públicos pasaron a disponer del listado de segurados del sistema ISAPRES. La otra explicación, más probable, es que los copagos en el subsistema privado constituyen un elemento moderador eficaz, elemento que no existe en el subsistema público.

\section{UNA PERCEPCIÓ N DE LOS DILEMAS Y PERSPECTIVAS DEL SISTEMA DE SALUD EN CHILE}

\subsection{Dilemas}

Los dilemas básicos del Sistema deSalud en Chile se pueden identificar como:

- Una clara definición del Mix Público Privado. Conforme hemos podido analizar, el subsistema privado que se ha desarrollado a partir de 1980 fue construido en base a fuertes subsidios públicos que sólo recién se buscan eliminar. La evolución de la participación privada va depender de dos variables con direccionalidad antagónica. Por una parte la mencionada eliminación de subsidios y esclarecimiento de los usuarios, que tenderá a disminuir la importancia de la atención privada; por outro lado, el crecimiento de los ingresos de la población, lo que tiende a permitir que una parte mayor de la población tenga acceso a planes de salud privados más atractivos.

- El diseño del Rol Regulador del Estado. El Estado Chileno, clásico y efectivo prestador de salud desde la Reforma de 1952, tiene ahora que asumir un rol netamente regulador, lo que conlleva un cambio cultural acentuado que como se sabe es muy difícil para las estructuras públicas tradicionales.

- La competitividad de los Servicios de Salud. Los servicios (regionales) de Salud, efectivos prestadores de servicios en la realidad presente y futura del Sistema Chileno, se ven lanzandos en una realidad competitiva con los servicios privados dentro de la cual tienen que demostrar no solamente su costo-efectividad como su capacidad de respuesta a las expectativas de prestadores y usuários.

- La Satisfacción Usuaria y Satisfacción de los Prestadores.

Los diferenciales hoy día existentes hacen que se pueda imaginar que los servicios públicos puedan mantener un grado competitivo de satisfacción de esos dos aspectos clave para el éxito del sistema.
- La solución de las Listas de Espera.

Como en los sistemas públicos de la mayoría de los países, las listas de espera para determinados tipos de enfermedades, son un problema actual en Chile (las colecistectomías han sido un ejemplo frecuente).

- La Diferenciación de los Estabelecimientos y Opciones de Atención.

Como en la mayoría de los países de Latinoamérica, el sistemas chileno todavía no se ha diferenciado suficientemente mejores en formas costo-efectivas de atención, como la atención domiciliaria, el hospital día, la cirugía ambulatoria, las casas de atención de enfermería, entre otras. El nuevo perfil nosológico que ya se delinea el en País, exige tales formas alternativas de atención.

- La Frontera entre los Servicios de Salud y la Actividad Social y Comunitaria en general.

En la medida que una proporción elevada de la clientela de los servicios es de edad avanzada, resta el dilema de la división de atribuciones entre la atención de salud y la atención social en general (atención social en general (atención al anciano noenfermo), lo que no es fácil en realidades como la chilena sin una estructura de apoyo social muy desarrollada.

\subsection{Perspectivas}

El futuro de ese sistema, en lo relacionado al mix público-privado, es enfrentado por el Ministerio de Salud de una forma que permite encarar sus resultados con cierto optimismo. Hay algunos indicadores de estabilización momentánea de la aplicación del rol del sector privado. Por ejemplo, desde que los hospitales públicos disponen de los listados de afiliados y pasan a exigir los co-pagos correspondientes, se observa en algunos Sistemas Regionales una estabilización de la afiliación a ISAPRES. Ese fenómeno es atribuido además a los cambios en la legislación, lo que resultó que esas instituciones se mostraron menos agresivas en la captación de nuevos afiliados. De hecho, la incorporación de nuevos afiliados incorporados al año en relación al total de beneficiarios ha bajado de un auge histórico de 24\% en 1990 hacia los 2,6\% en $1995^{6,7}$. En otros puntos del Sistema, (Servicio de Salud Metropolitano Central) se llega a observar una cierta relación de inversión de lo privado a lo público (en general trabajadores de bajo ingreso que perciben que no lograrán cubrir los co-pagos si se mantienen afiliados al subsector privado). Algunos servicios regionales de salud (ejs: Viña del Mar, Valparaíso, Coquimbo y otros) desarrollan a su vez propuestas 
de mejora de gestión a niveles de sus hospitales, lo que resulta en la captación de importante proporción de la clientela privada (el parque hospitalario privado fuera de Santiago es, en general, bastante escazo).

Aún en el área metropolitana, algunos hospitales como el Calvo Mackenna, disponen de propuestas de gestión bastante ágiles, lo que los torna muy competitivos en algunos nichos de mercado (en este caso, la cirugía cardiológica infantil).

En la medida que se den las condiciones políticas para que el Ministerio de Salud profundice su rol rector, que instituciones de gran potencialidad, como la Superintendencia de ISAPRES, acentuen su carácter regulador sobre el sector privado y los servicios regionales de salud prosigan con sus proyectos de modernización, la perspectiva del sector público de salud en Chile puede ser muy positiva.

\section{CONCLUSIONES}

Esa perspectiva optimista del Sistema de Salud de Chile es apoyada por algunas características que vale la pena resaltar:

- Se trata de un Sistema con robusto y tradicional componente público en acentuado proceso de modernización.

- La cobertura de servicios obedece, a una rigurosa lógica geográfica (áreas adscritas a cada Servicio Regional y a cada municipalidad).

- En consecuencia, dicha cobertura obedece, de igual forma, a rigurosa lógica demográfica. En el caso de atención primaria se busca agregar progresivamente un componente de "competencia administrada" representado por la inscripción voluntária al Consultorio local que le convenga al cliente.

- Hay una cobertura amplia de servicios, incluso en áreas rurales aisladas donde se utilizan postas conducidas por personal no-médico, apoyado por visitas periódicas de personal profesional (médico, matrona y enfermera). A esos niveles, se observa una participación social muy activa, que además se empieza a observar en de los servicios urbanos, básicos y hospitalários, con los Comités de Desarrollo de Servicios (El primer Encuentro Nacional de Consejos de Desarrollo, reunió en Marzo de 1996 a 59 entidades del tipo $)^{13}$.

- Se observa mecanismo estable de financiamiento de servicios (basado en el descuento exclusivo de $7 \%$ en los sueldos). El reparto de eses recursos a los Servicios se realiza desde 1995, bajo mecanismos innovadores y diversificados (financiamiento per cápita en atención primaria, pago asociado a diagnóstico y pago por procedimientos especiales, en atención secundária y terciária). Finalmente, se busca atender a la lógica global de las acciones mediante un Compromiso de Gestión firmado entre los Servicios Regionales y las instituciones financiadora y rectora (Fonasa y Ministério de Salud) y que además se desgloza en compromisos entre esos Servicios Regionales y sus establecimientos (hospitales y unidades básicas de salud consultorios).

- Chile dispone de un plantel de recursos humanos bastante calificados y que, además, en su promedio exhibe un invidiable nivel de compromiso social.

- Las condiciones mencionadas estimulan al desarrollo de propuestas de producción de servicios y de gestión muy creativas a lo largo de todo el País (gestion estratégica, gestión de calidad, re-inginiería, entre otras).

- Como política de gobierno y de salud hay un considerable estímulo a la participación social lo que se concreta incluso por los mencionados Consejos de Desarrollo de establecimientos de salud.

\section{ANEXO \\ 6. ASPECTOS DEL DESARRO LLO DEL SISTEMA DE SALUD DE CHILE Q UE PUEDEN INTERESAR PARTICULARMENTE A OTRO S PAÍSES DE LATIN O AMERICA}

Del desarrollo de la cooperción OPS en Chile, se destacan algunos temas que pueden tener mucho interés para otros países Latinoamericanos.

- Organización y Gestión de Servicios Regionales de Salud: Algunos Servícios Regionales de Salud (SRS) en Chile se encuentran desarrollando propuestas de gestión muy innovadoras, con utilización intensiva entre otras de Gestión Estratégica, y Gestión de Calidad (Ej. SRS VaparaísoSan Antonio; SRS Viña del Mar-Quillota, SRS Metropolitano Central, SRS Ñuble, entre otros).

- Metodología innovadora de remuneración de servicios de salud. En este caso, se encuentran el pago per cápita para la atención primaria, el pago asociado a diagnóstico (PAD), los Compromisos de Gestión FONASA/Servicios y Servicios/Establecimientos, las iniciativas de financiamiento de trasplantes y más recientemente, las licitaciones de listas-de-espera.

- Sistema de Vigilancia Sanitaria, especialmente el registro y fiscalización de medicamentos.

- Reglamentación estatal de los Seguros Privados de Salud. La Superintendencia de ISAPRES. 


\section{REREN CIAS}

1. CENTRO DE ESTUdiOS PÚBLICOS. Estudio Nacional de Opinión Pública $N^{o} 1$. Tercera Serie. Nov.l Dic. 1994. Doc. de Trabajo No 227. CPU. Santiago. Enero 1995.

2. HORWITZ, A. Servicio nacional de salud y la salud pública chilena In: La salud en el proceso de desarrollo chileno. Santiago, Minsal/OPS. 1995. p. 63-83.

3. HORWITZ-FELIU, V. El sistema de salud chileno: um problema pendiente. Puntos de Referencia, (Santiago) 123: $1-13,1993$

4. HORWITZ, N. et al. Salud y estado en Chile: organización social de la salud publica. Santiago de Chile, OPS, 1995.

5. ILLANES, M.A. et al. História social de la salud pública en Chile. 1880-1973. Santiago. 1993. (Colectivo de Atención Primaria).

6. VARIACIÓN neta de los beneficiarios en el sistema ISAPRES año a año. La Epoca. Santiago, 26 Feb.96. p.19.
7. MINISTERIO DE SALUD Fuerza de Tarea Participación Social Logros y avances en participación social en 1995: consejos de desarrollo. Santiago, 1996.

8. MINISTERIO DE SALUD. Atención de salud en Chile. Santiago. 1995. (Cap. 1: Situación Actual y Perspectivas de la Salud en Chile).

9. MONTT, P.L. \& SERRA, P.B. La descentralización educativa en Chile: el traspaso de la educación a los municipios. Santiago, CEPAL, 1994.

10. NACIONES UNIDAS. Comisión Económica para América Latina y el Caribe. Panorama social de América Latina 1995. Santiago de Chile, 1995.

11. ORGANIZACION FOR ECONOMIC COOPERATION AND DEVELOPMENT. The reform of health care: a comparative analysis of Seven OECD Countries. OECD Pub. Paris. 1992.(Health Policy Studies No 2).

12. SUPERINTENDENCIA DE INSTITUCIONES DE SALUD PREVISIONAL. Bol. Estad. Ene-Dic. 1995. Santiago. 1995. 